\title{
Performance Improvement of Stream-Centered Probabilistic Matrix Factorization Method Using Weighted Reservoir Sampling and Parallel Computing
}

\author{
Berman Danyel Sinaga and Saiful Akbar \\ School of Electrical Engineering and Informatics, ITB, Indonesia \\ Ganesha 10, Bandung 40132, Indonesia \\ berman.danyel@gmail.org,saiful@informatika.org
}

\begin{abstract}
Application of the recommender system on the online platform turns out to produce a large volume of data and at an unexpected rate, making it more realistic to study the recommender system under streaming settings. Many studies have tried to develop a streaming recommender system, one of which is Stream-centered Probabilistic Matrix Factorization (SPMF). In SPMF, model will be updated continuously every time a data stream window enters the system using the data managed in reservoir plus the data in data stream window. This study focuses on developing a reservoir sampling technique in SPMF method, namely uniform random sampling, which can result in model ranking errors due to the loss of observational data from memory. To reduce this consequence, sample is selected based on the matrix factorization score, which is considered to represent the popularity of item. In addition, this research also developed a parallelization technique for the updating of the SPMF algorithm for the purpose of increasing scalability. Based on the experiments conducted on E-commerce dataset, the proposed method is proven to have better recommendation quality than the baseline method and reaches at least $84 \%$ quality of BPR-MF with a reduction of the dataset up to $78 \%$ and computation time 60 times faster. Parallel computing can also increase computation speed by $23 \%$ by using 4 workers.
\end{abstract}

Keywords: Recommender System, Parallel Computing, Data Stream, Reservoir Sampling

\section{Introduction}

Application of recommender systems on online platforms such as eBay and Netflix has resulted in large volumes of data and at an unexpected rate. For example, more than 10 million transactions per day were made on eBay and Netflix gained more than 3 million new subscribers from mid-March 2013 to April 2013. The data is continuous, high-velocity and time-varying which is the nature of the data stream. Therefore, it will be more realistic to study the recommender system using a streaming data framework[1].

According to [1] recommender system under the streaming setup should be able to tackle the following challenges simultaneously. First, the unknown size. New users and new items enter the system continuously so that the number of users and items is never known. Most of the algorithms that exist today assume that all data is available. Second, real-time updating. One of the characteristics of the data stream is high speed. Therefore the recommender system needs to update the model instantly to capture the instant desire of the user. Third, concept shift. Item's popularity and user preferences change over time. Recommender system must be able to pick up on these signals and adjust them in a timely manner.

There are many related studies have tried to solve the challenges in the previous paragraph. [1] and [2] proposes an online model update every time new observations enter the recommender system. Unfortunately, the strength in processing time achieved by the online approach has a disadvantage in terms of predictive quality. The main problem with the online approach is that the model tends to remember only recent activities (short-term memory) so that long-term preferences cannot be maintained. [3] maintain a random sample from the coming data and update the model only based on the sampled instances. Their aim is to stop the system from forgetting users' long-term interests. But according to [4], this sampling strategy tends to overlook users' drifted interests as it has smaller probability of sampling to more recent data.

Received: September $7^{\text {th }}, 2020$. Accepted: September $15^{\text {th }}, 2021$

DOI: $10.15676 /$ ijeei.2021.13.3.8 
The latest research that tries to solve the problem of the streaming recommender system with a reservoir sampling approach is [4] namely streaming probabilistic matrix factorization (SPMF). In this study, the model update will be carried out after a certain amount of data in a certain time is entered into the system (window). The input used to update the model is the data stored in the reservoir plus the last window. The goal is for the model to capture user drift interest while maintaining long-term user preferences. SPMF produces better quality recommendations than previous method. However, the selection of reservoir data samples at SPMF is still inaccurate. The selection of reservoir data samples was carried out using uniform random sampling, which could potentially lead to a ranking error in the BPR model. This will result in a decrease in the prediction quality of the model, especially the long-term interest of the user. In addition, the reservoir approach proposed by previous researchers is also not scalable where at high data stream rates the model cannot maximize the quality of recommendations. Based on the remaining problems in the previous paragraph, this study tries to improve the reservoir data sampling technique at SPMF by adding weighting the sample based on item popularity. With this weighting, it is hoped that the data that are not selected in the reservoir are unpopular data so that they can be considered as negative items. In addition to increasing scalability, the recommender system is designed in such a way that model updates can be computed in parallel. This parallel computation is also expected to provide room for additional reservoir size without causing overload.

This paper is organized as follows: Section 2 provides preliminary concepts about recommendation model and parallelization matrix factorization. Section 3 formulates streaming recommendation problem. Section 4 describes our proposed method. Section 5 and 6 discusses the experimental evaluation of our proposed method. Existing research related to our work is surveyed in Section 7. Section 8 discuss about contribution of research in real life. Section 9 closes this paper with some conclusive remarks.

\section{Preliminary}

In this section, we will explain the algorithms from related studies which are the reference in developing the algorithms proposed in this work.

\section{A. Bayesian Personalize Ranking}

Based on the type of feedback in the dataset, the recommender system can be divided into 2 groups, namely explicit feedback (e.g. ratings) and implicit feedback (e.g. searches, purchases, clicks etc.). Bayesian Personalize Ranking (BPR) is a recommender system method with implicit feedback. BPR is designed for optimization of personal item ranking. Let $U$ be the set of all users and I the set of all items. The available data are implicit feedback $S \subseteq U \times I$ (left side of Figure 1). The task of the recommender system is to present the total ranking $>_{u} \subset I^{2}$ of all items personally to all users.
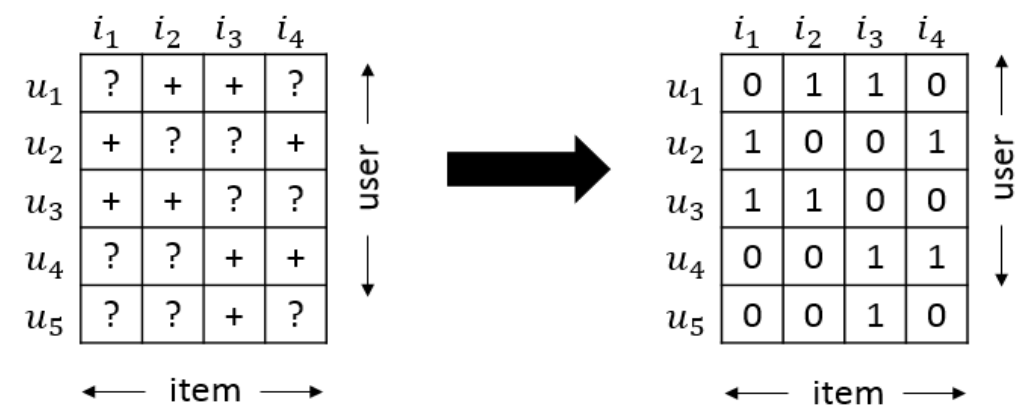

Figure 1. User-item Matrix With Implicit Feedback

In implicit feedback, the items observed by the user are considered as positive classes. while unobserved items can be negative class or missing value. In general, the recommender system will calculate an $X_{u i}$ score which implies the user's interest towards item. Then the items are 
ranked in order of scores. The machine learning approach to recommender systems generally uses only positive labels as training data. However, BPR uses a different approach, namely using a pair of positive items and negative items as input for ranking optimization. The user-item pairs in Figure 1 are reconstructed into item-item pairs for each user. If the item is observed by user $u$, it is assumed that the item is preferred by the user over the item that is not observed. For example in Figure 2 user $u_{1}$ has historical activity with $i_{2}$ but not $i_{1}$, so it is assumed that user $u_{1}$ prefers $i_{2}$ over $i_{1}$. On items that are both observed and unobserved by the user, no relationship can be made.

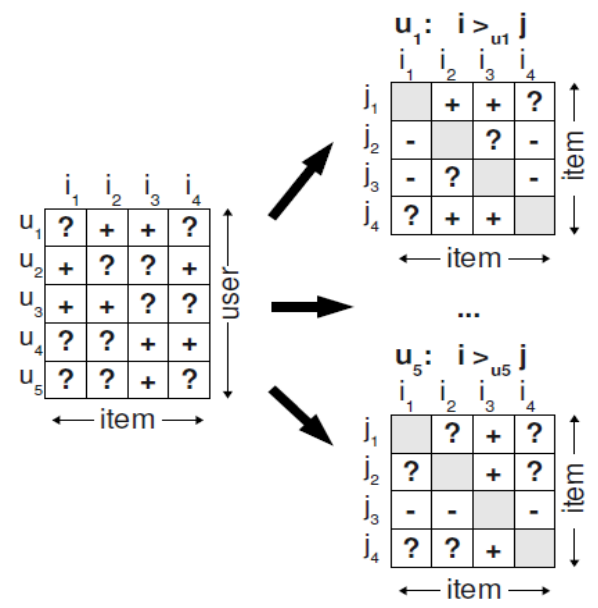

Figure 2. Item Relationship Matrix at BPR

In the following we rewrite how BPR was derived in the work of Rendle et al [5]. Ranking optimization on BPR is derived from the Bayesian analysis which can be written as:

$$
p\left(\theta \mid>_{u}\right) \propto p\left(>_{u} \mid \theta\right) p(\theta)
$$

where $>_{u}$ is preference structure for user $u$ as depicted in Figure 2. On the right side of the figure, $+\operatorname{sign}$ indicates that user $u$ prefers item $i$ to item $j$. The individual probabilities that the user actually prefers item $i$ to item $j$ are:

$$
p\left(i>_{u} j\right):=\sigma\left(x_{-} u i j(\theta)\right)
$$

where $\sigma$ is the sigmoid function:

$$
\sigma(x):=\frac{1}{1+e^{-x}}
$$

In this case $x_{u i j}(\theta)$ is a function that models the relationship between user $u$, item $i$ and item $j$ which can be decomposed into:

$$
x_{u i j}=x_{u i}-x_{u j}
$$

Because the above framework is general, we can apply collaborative filtering such as a factorization matrix that can predict interactions between users and items.

In brief, the complete form of the maximum posterior probability optimization (BPR-Opt) that has been discussed in the previous paragraph can be determined as:

$$
\begin{aligned}
B P R-O P T & :=\ln p\left(\theta \mid>_{u}\right) \\
& =\ln p\left(>_{u} \mid \theta\right) p(\theta) \\
& =\sum_{(u, i, j) \in D_{S}} \sigma\left(x_{u i j}\right)-\lambda_{\theta}\|\theta\|^{2}
\end{aligned}
$$


The optimization algorithm used is LEARNBPR, namely the SGD algorithm with triple training bootstrap sampling $(u, i, j)$. The BPR-OPT gradient to model parameters is

$$
\begin{aligned}
\frac{\partial B P R-O P T}{\partial \theta}=\sum_{(u, i, j) \epsilon D_{s}} \frac{\partial}{\partial \theta} \ln \sigma\left(\check{x}_{u i j}\right)-\lambda_{\theta} \frac{\partial}{\partial \theta}\|\theta\|^{2} \\
\propto \sum_{(u, i, j) \epsilon D_{s}} \frac{-e^{-\check{x}_{u i j}}}{1+e^{-\check{x}_{u i j}}} \cdot \frac{\partial}{\partial \theta} \check{x}_{u i j}-\lambda_{\theta} \theta
\end{aligned}
$$

and

$$
\frac{\partial}{\partial \theta} \breve{x}_{u i j}=\left\{\begin{array}{cc}
\left(h_{i f}-h_{j f}\right) & \text { if } \theta=w_{u f} \\
w_{u f} & \text { if } \theta=h_{i f} \\
-w_{u f} & \text { if } \theta=h_{j f} \\
0 & \text { else }
\end{array}\right.
$$

\section{B. Weighted Random Sampling Over Data Streams}

In this section, we describe weighted random sampling over data streams as explain in [6]. Random sampling is used to select $m$ samples from a population of size $n$. If all items have an equal probability of being selected, the problem is called uniform random sampling. If the probability of each item depends on the weight of the item, the problem is called weighted random sampling (WRS).

There are several algorithms that can be used to solve the problem of weighted random sampling over data streams, but we prefer to focus on the reservoir approach as we did to solve the streaming problem. There are 2 main algorithms that can be used, namely General Purpose Unequal Probability Sampling Plan of Chao [7] (we will call A-Chao) and Weighted Random Sampling with a Reservoir algorithm of Efraimidis and Spirakis [8] (we will call A-ES). In AChao, when a new item is checked, its relative weight is calculated and used to determine whether the item will be included in the reservoir or not. If yes, then one item from the reservoir will be randomly selected to be removed from the reservoir. While in A-ES, each item independently generates a random number $u_{i} \in(0,1)$ which is then used to calculate key $k_{i}=u_{i}^{1 / w_{i}}$. New items are calculated by the same procedure and compared with the smallest key in the reservoir. If the new item's key is greater, then the new item will replace the item with the smallest key in the reservoir.

Among two available options, we do not really focused on choosing the best algorithm, but we are focused more on how to calculate the weights according to the challenges of a recommender system with a streaming framework.

\section{Distributed Stochastic Gradient Descent}

One solution to increase the speed performance of machine learning models is parallel or distributed computing. Distributed stochastic gradient descent (DSGD) is an optimization algorithm designed to work in parallel or distributed on a matrix factorization. This algorithm is developed from stochastic gradient descent (SGD). The challenge with SGD distribution is that every single update on SGD depends on the previous step. For example in SVD, the single SGD update is

$$
\theta_{n+1}=\theta_{n}-\epsilon_{n} \hat{L}^{\prime}\left(\theta_{n}\right)
$$

which means that to calculate $\theta_{n+1}$ the value of $\theta_{n}$ must be known first. This makes it difficult for SGD to be computed in parallel or distributed. But in the case of matrix factorization the SGD process has an exploitable structure. Training points $z_{1}=\left(i_{1}, j_{1}\right)$ and $z_{2}=\left(i_{2}, j_{2}\right)$ will be independent as long as $i_{1} \neq i_{2}$ and $j_{1} \neq j_{2}$. Likewise, the block matrix $Z^{i j}$ will be mutually independent if there are no rows and columns that are shared by the matrix block. A set of independent matrix blocks is called a strata. 

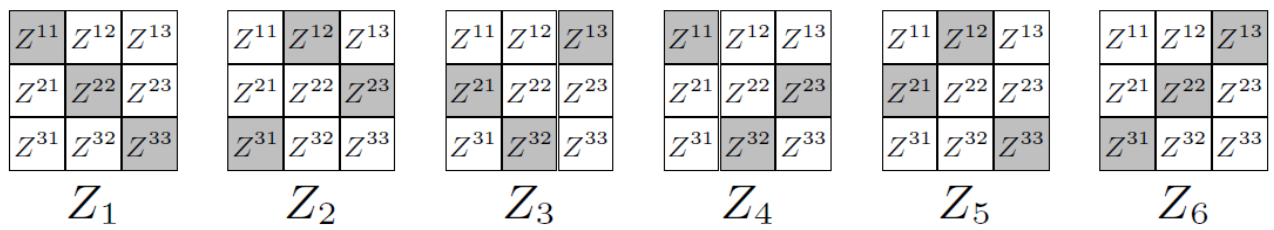

Figure 3. Stratum on $Z$ Matrix With $3 \times 3$ Blocks

Blocks at the same stratum can be processed in parallel and distributed. The global loss function $L(\theta)$ can be calculated from the sum of the loss function $L_{S}(\theta)$ in each matrix block

$L(\theta)=w_{1} L_{1}(\theta)+w_{2} L_{2}(\theta)+\cdots+w_{n} L_{n}(\theta)$

To run DSGD, first the matrix is partitioned into $d \times d$ matrix blocks with the respective sizes $(m \times d) \times(n \times d)$ as shown in Figure 3. Then run SGD in parallel on each block in the selected strata. After one stratum is finished, then move on to the next stratum.
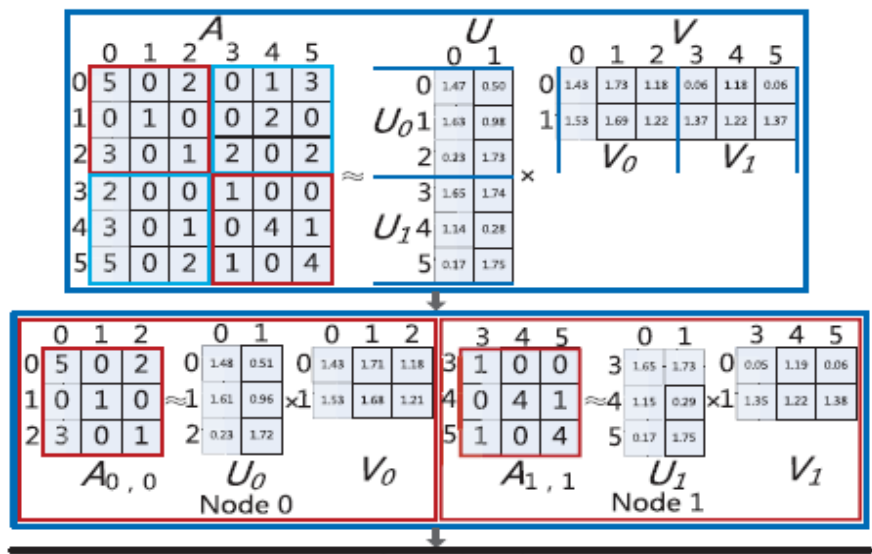

Synchronization

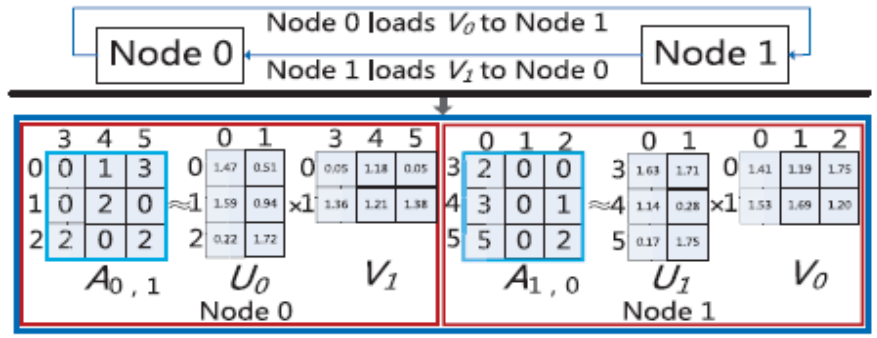

Figure 4. DSGD Ilustration

\section{Problem Statement}

\section{A. Reservoir Sampling Impact}

Selection of reservoir data samples at SPMF was carried out uniformly randomly following the proposed reservoir sampling technique [9] .This sampling technique will indeed produce samples that have the same global item distribution as the data population. However, it should be noted that in reservoir sampling, data points that are not retrieved will be discarded from memory and considered as negative items when running LEARN-BPR optimization. If the item that is not taken is an item with a small $x_{u i}$ score, it will not be a problem because the smaller the $x_{u i}$ score means the user doesn't like the item. On the other hand, if the items that are not picked up are items with a high $x_{u i}$ score, there will likely be a ranking error. The greater the $x_{u i}$ score that is not taken, the greater the chance of a ranking error. 
Suppose that in user score which implies the order of items based on the $x_{u i}$ score from highest to lowest is a, c, d, e, b. When item a is not selected during reservoir sampling, any iteration involving item a will inevitably result in an optimization error. However, if the item that is not selected is item $\mathrm{d}$, then the chance of an optimization error is $50 \%$ when paired with item e or b. And if the item that is not selected is item $b$, there will be no optimization error assuming the order of popularity of the items mentioned is in accordance with the ground truth. From equation II.10 it can be seen that every SGD step in a BPR will involve user factor $u$, item factor $i$ and item factor $j$. Therefore, optimization errors as described above impact not only the users involved, but also other users.

\section{B. Scalability}

To ensure that the streaming recommender system can work in real time, it is necessary to ensure a balance between computing capacity and streaming data rates. If the streaming data rate exceeds the compute capacity, the system becomes overloaded. For example, it takes 2 seconds to process each data input in the recommender system while the streaming data rate is 1 activity per second. Since the compute resources can only process 1 activity every 2 seconds, queues become pile up and response times increase due to queue waiting times.

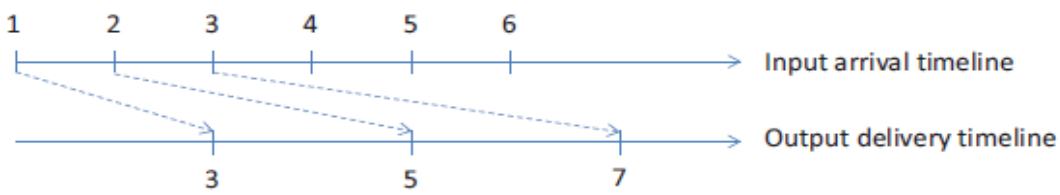

Figure 5. Overload Scenario

In SPMF the overload problem is resolved by stopping model training when a new window arrives. This means that the model training process can be considered complete even though the objective function has not converged, for example in a condition where the data stream rate is very high. As a result, the quality of recommendations is not optimal. In addition, one of the issues in the reservoir sampling approach is that the quality of the predictions depends on the size of the reservoir. The bigger the reservoir size, the better the prediction quality. However, increasing the reservoir size will certainly increase the complexity of the algorithm and memory usage. To increase scalability, the LEARN BPR algorithm will be run in parallel. The LEARN BPR parallelization technique was adapted from DSGD.

\section{Proposed Method}

\section{A. Model Updating Algorithm Based on Input Data Stream}

When handling incoming data streams consistently, people generally want to avoid the costs of retraining the model every time a new data instance arrives. The recommender system designed in this study adopts incremental learning. The model is updated every time the data stream window enters the system. There are 2 main processes in the design recommender system, namely updating the model and reservoir sampling. The model update aims to update the parameters in the user matrix and item matrix by running the LEARN-BPR algorithm using the data in the reservoir and the latest window as input. Meanwhile, reservoir sampling aims to keep the data size constant due to memory limitations so that data not selected during reservoir sampling will be removed from memory. Reservoir sampling is carried out after the model update process is complete and this data will be used in the next updating process. Reservoir data selection was carried out using weighted random sampling techniques. Each data point in the reservoir and window is ranked descending based on the multiplication of the matrix decomposition. Then the weight of each data point can be calculated using:

$$
p(i \mid u) \propto \exp (-r(i \mid u) / \lambda), \lambda \in R+
$$

where $r(i \mid u)$ is ranking of item i according to user $u$ which is calculated based on the matrix factorization score. 
Input: The current set of parameter $\Theta^{t}=\{U, V\}$

the current reservoir $R=\left\{s_{1}, s_{2}, \ldots, s_{|R|}\right\}$,

a window of new data streams $W=\left\{e_{t+1}, e_{t+1}, \ldots, e_{t+|W|}\right\}$;

Output: The updated latent vectors $\Theta^{t+|W|}$ for users and items;

1

while BPR-Opt is not convergen do

2

Sample a data instance $x_{u i}$ from $\{R \cup W\}$ randomly;

3

Sample a negative examples $x_{u j}$ from $D_{t}^{-}=D-R-W$;

4

$\theta \leftarrow \theta+\eta\left(\frac{-e^{-\breve{x}_{u i j}}}{1+e^{-\check{x}_{u i j}}} \cdot \frac{\partial}{\partial \theta} \check{x}_{u i j}-\lambda_{\theta} \theta\right)$

5 end

6

foreach data instance $\left\{u_{i}, v_{j}\right\} \in\{R \cup W\}$ do

7

Get $u_{i}$ or $v_{j}$ from $\Theta^{t}$;

8

Compute the score $\left(u, i, \Theta^{t}\right)=u_{i} \cdot v_{j}$

\section{9}

Rank data instances decreasingly according to $f\left(u, i, \Theta^{t}\right)$

10

Compute $p(i \mid u)$ from equation 10

\section{1 end}

12

Sample $|R|$ data instance $x_{u i}$ from $\{R \cup W\}$ based on $p(i \mid u)$

13

Replace old reservoir

\section{B. LEARN-BPR Parallel}

Considering that learning at BPR is pairwise learning, the direct implementation of DSGD in BPR LEARN will result in bias in ranking items that are in different blocks. As an illustration, suppose Figure 6 is a $2 \times 2$ slice of a matrix block. Then we take an example of optimization for one user, for example user $\mathrm{u}_{1}$. In block $Z_{1}$, item $i_{1}$ can only use items $i_{2}$ and $i_{3}$ as pairs and in block $Z_{1}$, items $i_{5}$ can only use items $i_{4}$ and $i_{6}$ as pairs. Therefore, the model will not know how the ranking of item $i_{1}$ against items $i_{4}$, and $i_{6}$ or the ranking of items $i_{5}$ against items $i_{2}$ and $i_{3}$.
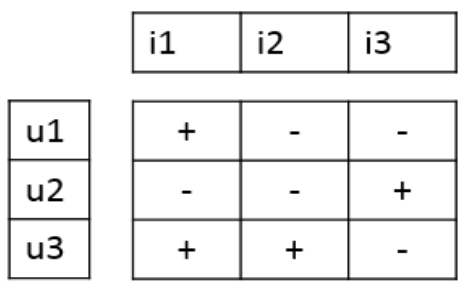

u3

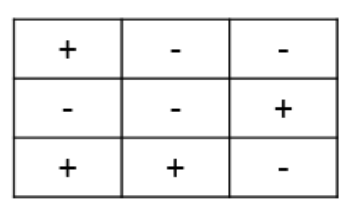

$Z_{1}$
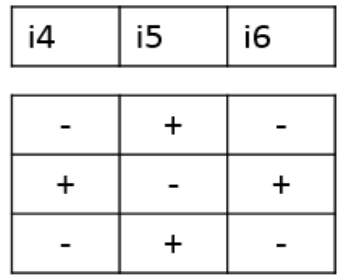

$Z_{2}$

Figure 6. Illustration of Item Pair on Block matrix 
One solution to enable item optimization between blocks is to exchange / permutate items between blocks. Suppose that at epoch 1 the position of the item is as in Figure 6. Then on the next epoch all items will be randomly rearranged in both blocks and so on. Matrix factorization on each block using stochastic gradient descent the same as in DSGD. Additional computational burdens include: synchronization, permutation and division of the matrix into matrix blocks. Here is the LEARN BPR parallelization algorithm.

Input: $Z, U O, V 0$, block size $d$

Output: Item matrix and user matrix

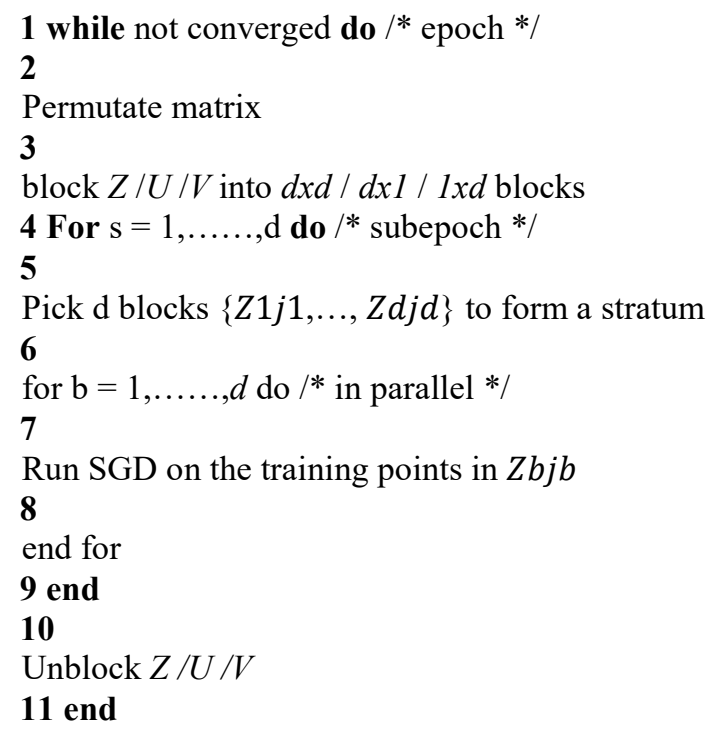

\section{Experimental Setup}

A. Data

Experiments in this study used 2 datasets from different applications, namely MovieLens and E-commerce. MovieLens dataset contains 100,000 ratings on a scale of 1-5 from 943 users on 1682 items. Because the recommender system algorithm used is implicit feedback, the rating score is ignored. So task of recommender system in this dataset is to predict the items that will be assessed by the user. Meanwhile, E-commerce's dataset contains 175,858 historical purchases and searches of 9,887 users on 3,713 items. Task of recommender system in this dataset is to predict which items the user will buy or search for.

In Figure 7 and Figure 8 the distribution of user activity is presented which is divided into 10 parts based on timestamps. The Movie Len dataset is dominated by new user activity for each part so that this dataset is expected to show how the performance of the algorithm designed to maintain the interest of old users. Meanwhile, the E-commerce dataset is dominated by longterm user activity for each part, so that this dataset is expected to show how the performance of the algorithm designed to capture user drift-interest (if any) while maintaining the user's longterm interest. As mentioned in section 1, capturing drift interest and maintaining user long-term interest is one of the challenges in the streaming recommender system. 


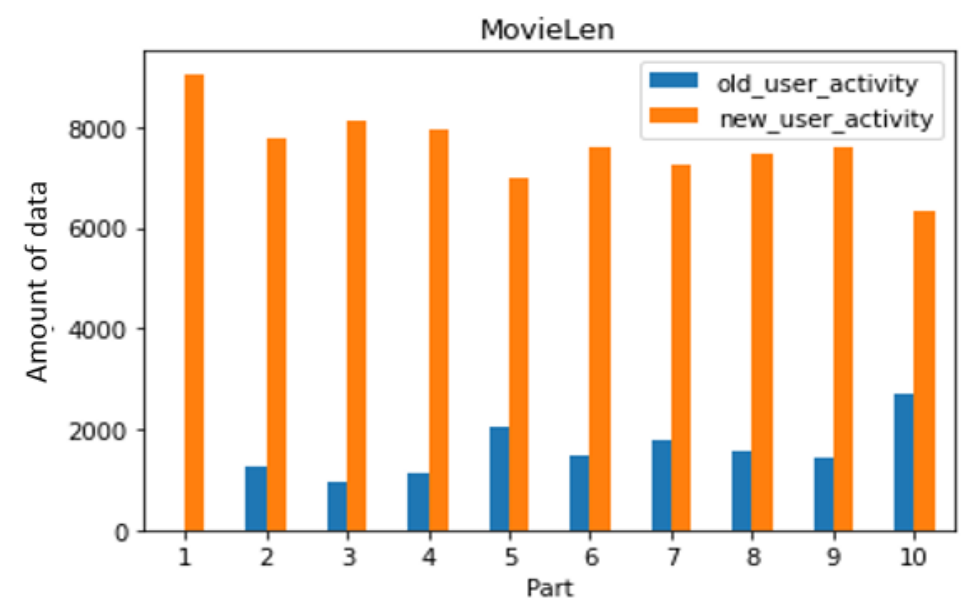

Figure 7. Distribution of user activity on the Movie Len dataset

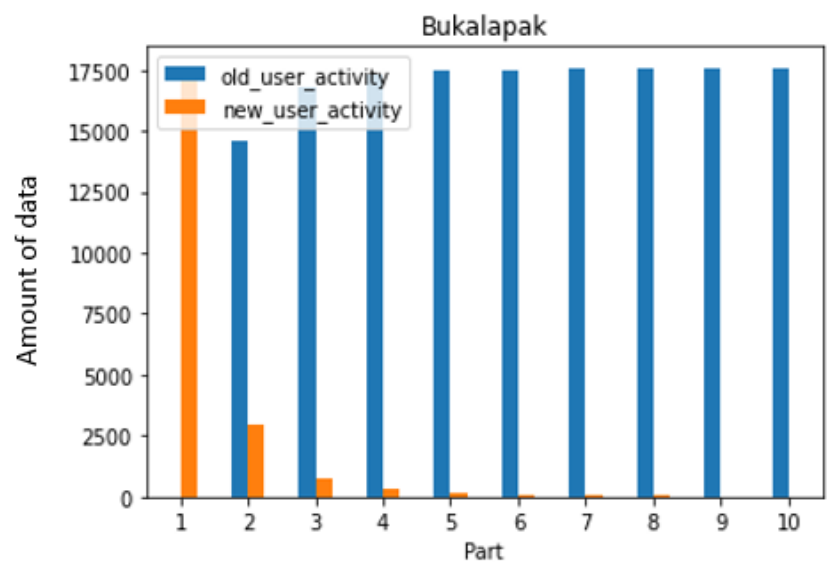

Figure 8. Distribution of user activity on the E-commerce dataset

\section{B. Evaluation Metric}

The metric used to measure the quality of the recommendations in this study is MAP (mean average precision). MAP is often used to measure the quality of task ranking on information retrieval (IR). Several studies regarding the top-N recommender system also use MAP as a metric such as in research [10] and[11]. To calculate the MAP for the top-N recommender system, we first determine the cutoff of the MAP. This cutoff is equal to $\mathrm{N}$ on top-N. In this study, the cutoff used is 40. Next is to calculate the Average Precision (AP) for each user. AP @ N can be calculated using the formula:

$$
A P @ N=\sum_{k=1}^{N} P(k) \Delta r(k),
$$

where $\Delta \mathrm{r}(\mathrm{k})$ is the change in recall from k-1 to k. Then MAP@ $\mathrm{N}$ is the average AP@ N of each user

$$
M A P @ N=\frac{1}{|U|} \sum_{u=1}^{\mid} U \mid(A P @ N)_{u}
$$

\section{Weighted Reservoir Sampling Experiment}

The WRS experiment was divided into 2 scenarios, namely the WRS- 1 and WRS- 2 scenarios. The WRS scenario uses the Movie Len dataset where the objective of this experiment is to measure the effect of the WRS method on the model's ability to maintain long-term memory. This is consistent with the historical characteristics of the Movie Len dataset where each time 
window is dominated by new users and new items. The test data is taken randomly without returning from a set of 10 observational data for each user and the rest is used as training data. Then the training data is divided into 2 equal parts. The first part is used as input for offline model training. Then the second part is further divided into 5 equal sections where these five sections will function as windows during the online training. Each window is tested using preselected test data.

Meanwhile, the WRS-2 scenario uses the E-commerce dataset where the objective of this experiment is to measure the effect of the WRS method on the model's ability to capture the concept shift while maintaining long-term interest. First of all the dataset is divided into 2 equal parts. The first part is used as input for offline model training. Then the second part is further divided into 5 equal sections where these five sections will function as windows during the online training. Each window is tested using the next window.

The method used as a comparison to the performance of the methods proposed in this scenario are as follows:

1. Bayesian Personalize Ranking - Matrix Factorization (BPR-MF)

How this method works can be seen in Section 2. During the evaluation it is assumed that all data is stored and can be used for model training. It is hoped that the performance of this offline method, with full access to historical data and unlimited time to update the model, will be the upper bound for the online approach.

2. Streaming Probabilistic Matrix Factorization (SPMF)

How this method works can be seen in Section 2. This method is a reference method in this research.

Reservoir size in this experiment is $|D| / 10$. To determine the hyperparameters, we first take the hyperparameters in the experiment conducted by Rendle [10] as a reference. Then we tried different combinations of hyperparameters with grid search and the following settings produce the highest MAP. Hyper parameters for the MovieLen dataset are: latent dimension factor $k=$ 50 , regularization $=0.01$, learning rate $\eta=0.1$ and geometric distribution parameter $\lambda=4.5$. Hyper parameters for E-commerce dataset are: latent dimension factor $k=150$, regularization $=$ 0.005 , learning rate $\eta=0.1$ and geometric distribution parameter $\lambda=20$.

\section{Parallel LEARN-BPR Experiment}

This experiment aims to see the effect of LEARN-BPR parallelization in terms of computation time and recommendation quality. In addition, this experiment will also show how the effect of permutation of item arrangement on the matrix on the quality of recommendations. The code implementation for Parallel BPR computation uses a multithreaded python module with 4 workers. There is no difference in algorithm complexity between parallel BPR and nonparallel BPR. The hardware used for computing in this scenario is an Intel ${ }^{\circledR}$ Core ${ }^{\mathrm{TM}} \mathrm{i}-3360 \mathrm{M}$ (a) 2.80 GHz CPU. The dataset used in this experiment is the MovieLen 20M dataset.

\section{Result and Discussion}

In Movie Len dataset, number of iterations for each window is $10^{5}$ iterations. Then every $10^{4}$ iterations measured the MAP value to see the gradient of the MAP function to the iteration. Meanwhile in the E-commerce dataset, the number for each window is $2 \times 10^{6}$ and the MAP value is measured every $10^{5}$ iterations.

\section{A. WRS-1 Scenario}

MAP as a function of iteration for the MRB and SPMF methods in the WRS-1 scenario is presented in Figures 9 and 10. 


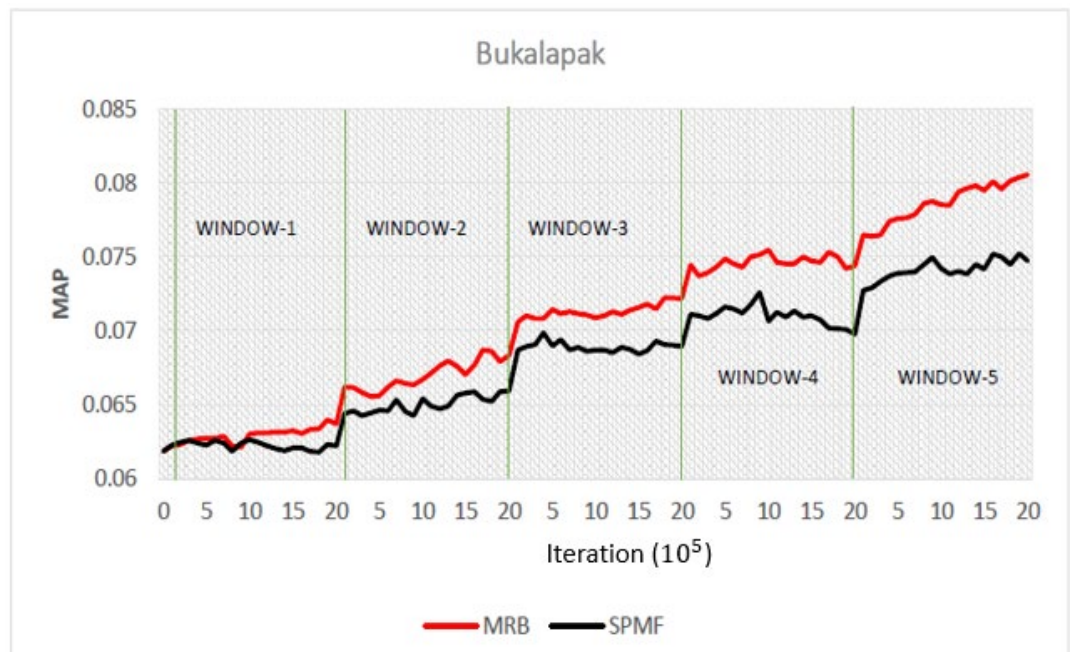

Figure 9. MAP as Function of Number of Iterations in WRS-1 Scenario In E-commerce Dataset

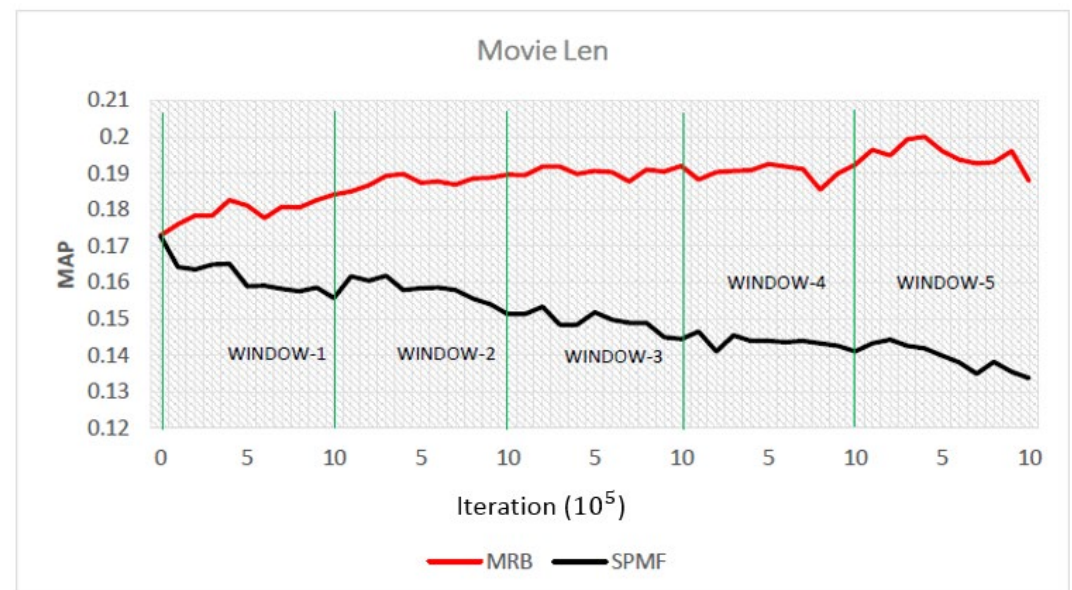

Figure 10. MAP as Function of Number of Iterations in WRS-1 Scenario In Movie Len Dataset

In Figure 7 above, it can be seen that the MRB method has an MAP value with a positive trend until it converges and there is an increase in the MAP value for each additional window, as well as the SPMF method. However, there is a gap in the MAP value between MRB and SPMF, the longer it gets bigger as the window increases. And in Figure 8, the MAP value of the MRB method still has a positive trend until it converges when the MAP value of the SPMF method continues to decrease as iterations are added. Because E-commerce dataset is dominated by old user activity for each window, each new window that comes in at least still has the same characteristics as the data in the reservoir so that the uniform random sampling technique is still good enough to select a sample that can sketch historical data even though it is not as good as weighted reservoir sampling technique so that at least the MAP value on the SPMF continues to increase for each additional window.

However, in the Movie Len dataset which is dominated by new user activity for each window, the SPMF uniform random sampling technique becomes very bad in selecting reservoir samples so that the learning process at SPMF is getting worse over time. This is because the uniform random sampling technique chooses samples randomly without paying attention to the results of the learning process that has been done previously. While the MRB method with the weighted 
reservoir sampling technique is designed to select a sample with a probability equal to the factorization matrix value which is the result of the previous learning process. In other words, the weighted reservoir sampling technique in the MRB method designed to work much better than the uniform random sampling technique at SPMF in selecting the most informative samples to be stored in the reservoir as a historical data sketch so that the model can maintain the preferences of old users both in terms of dataset characteristics which is dominated by old users and old users.

\section{B. WRS-2 Scenario}

WRS-1 scenario has been tested to see the ability of the method to maintain long-term user interest. Then in this WRS-2 scenario, it will be shown how the quality of the recommendations produced with a test scenario that is similar to the real world. The MAP graph as a function of iteration for the MRB and SPMF methods in the WRS-2 scenario is presented in Figure 11 and for the BPR-MF method is presented in Figure 12.

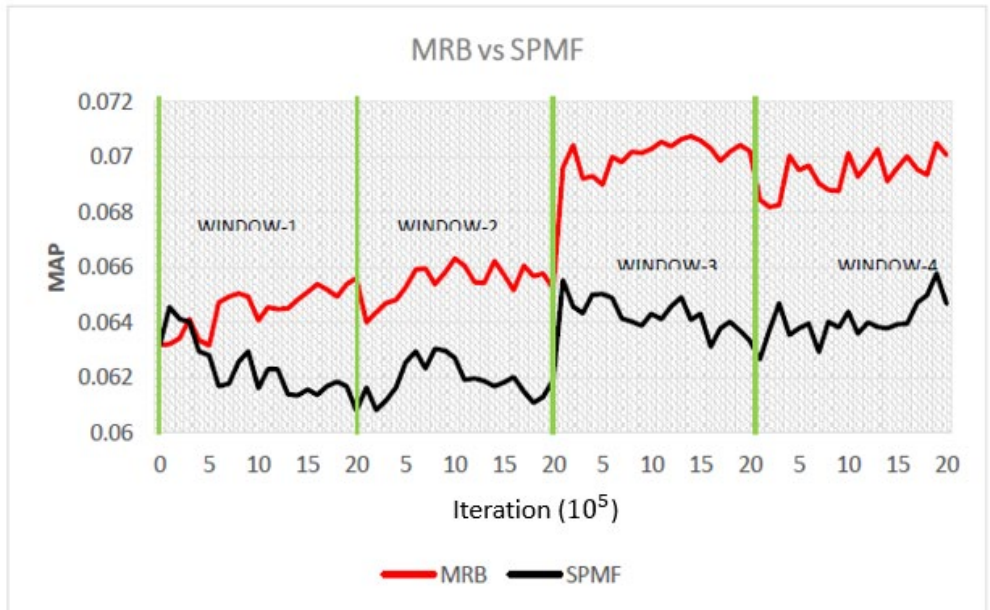

Figure 11. MAP as Function of Number of Iterations in WRS-2 scenario

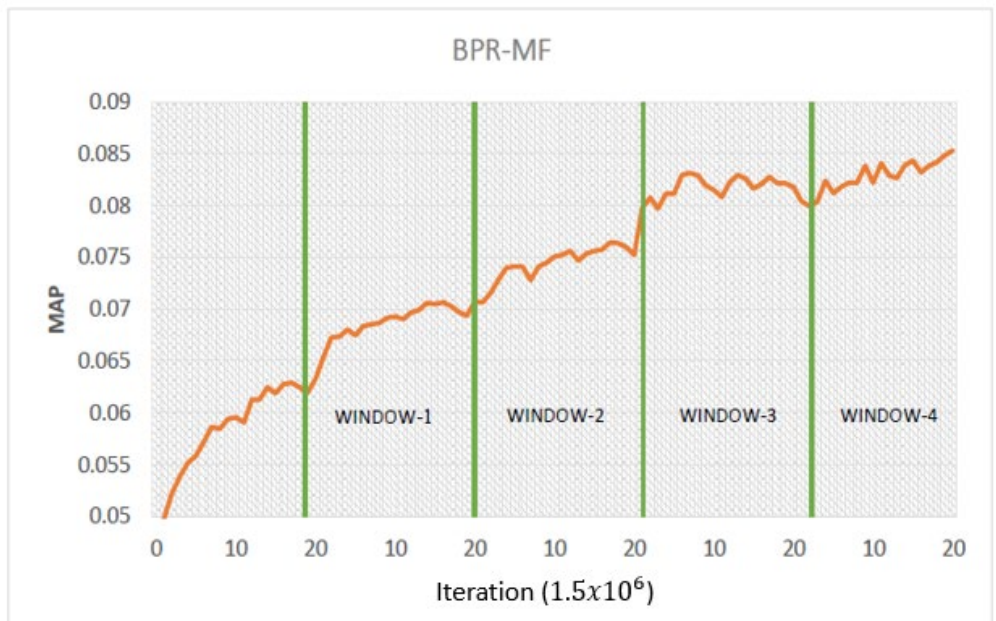

Figure 12. MAP as Function of Number of Iterations in BPR-MF Method

To see the final MAP value, the maximum MAP value is taken from each window. In Table 1 , the MAP value for each window is presented in the three methods tested. $\mathrm{w}_{0}$ is an analogy to 
the initial phase of the model when it was first run into production. Model $\mathrm{w}_{0}$ is trained using the data base training set.

Table 1. Comparison of MAP for each method in WRS-1 scenario

\begin{tabular}{|c|c|c|c|}
\hline Window & BPR-MF & SPMF & MRB \\
\hline $\mathrm{w}_{0}$ & 0.1802 & 0.1802 & 0.1802 \\
\hline $\mathrm{w}_{1}$ & 0.1857 & 0.1651 & 0.1841 \\
\hline $\mathrm{w}_{2}$ & 0.1963 & 0.1617 & 0.1897 \\
\hline $\mathrm{w}_{3}$ & 0.2039 & 0.1534 & 0.1920 \\
\hline $\mathrm{w}_{4}$ & 0.2072 & 0.1465 & 0.1925 \\
\hline $\mathrm{w}_{5}$ & 0.2169 & 0.1443 & 0.2000 \\
\hline
\end{tabular}

From Table 1 it can be seen that the MRB method has a better MAP value than the SPMF method for each window. This proves that the weigted reservoir sampling technique designed for the MRB method provides an increase in the final results of the recommendations. In addition, this also proves once again that the user's preferences in the real world are long-term as mentioned by previous researchers.

Compared to the BPR-MF method which is the upper limit of testing in this scenario, MRB has a fairly close MAP value. And when viewed from the computational speed side, the number of iterations needed to achieve a convergent MAP in MRB is $5 \times 10^{5}$ iterations and $3 \times 10^{7}$ iterations in BPR-MF is needed. Since there is no difference in the complexity of the algorithm for each iteration in the two methods, it can be said that MRB can significantly save computation time compared to BPR-MF which is 60 times faster. In Table 2 it can be seen that the MRB method reaches at least $83 \%$ of the quality of BPR-MF with a data reduction of $78 \%$.

Table 2. Relationship of Quality Recommendations and Dataset Reduction between MRB and BPR-MF Methods in the E-commerce Dataset

\begin{tabular}{|l|r|r|r|r|}
\hline \multicolumn{1}{|c|}{ Window } & \multicolumn{1}{l|}{$\begin{array}{l}\text { MAP } \\
\text { BPR-MF }\end{array}$} & \multicolumn{1}{l|}{$\begin{array}{l}\text { MAP } \\
\text { MRB }\end{array}$} & \multicolumn{1}{l|}{$\begin{array}{l}\text { Quality of MRB } \\
\text { against BPR-MF }\end{array}$} & Dataset Reduction \\
\hline $\mathrm{W}_{0}$ & 0.0632 & 0.0632 & $100 \%$ & $0 \%$ \\
\hline $\mathrm{W}_{1}$ & 0.0707 & 0.0656 & $93 \%$ & $67 \%$ \\
\hline $\mathrm{W}_{2}$ & 0.0764 & 0.0663 & $87 \%$ & $71 \%$ \\
\hline $\mathrm{W}_{3}$ & 0.0832 & 0.0708 & $85 \%$ & $75 \%$ \\
\hline $\mathrm{W}_{4}$ & 0.0853 & 0.0705 & $83 \%$ & $78 \%$ \\
\hline
\end{tabular}

\section{Parallel LEARN-BPR}

In this scenario there is one additional parameter that must be configured, namely the iteration block. The iteration block in question is the number of iterations in each matrix block prior to parameter synchronization. The choice of the number of iterations per block will affect the convergence rate and computation speed. To see the relationship between the number of iteration blocks with computation time and the rate of convergence, the parallel experiment was carried out 4 times with different number of iterations of blocks, namely $52.5 \mathrm{k}, 105 \mathrm{k}, 210 \mathrm{k}$ and $420 \mathrm{k}$ iterations. In Figure 13, MAP is presented as a function of the epoch to see how the number of iteration blocks is related to the convergence rate. From Figure 9 it can be said that the smaller the number of block iterations, the less the number of epochs needed to achieve convergence. 
This is because the algorithm certainly requires a certain number of permutations to achieve convergence. However, the problem is that the smaller the number of block iterations, the longer the computation time required for each epoch. This is because the smaller the iteration block, the more synchronization is done for each epoch. For a $52.5 \mathrm{k}$ block iteration configuration the number of synchronization for each epoch is 24 times, for a $105 \mathrm{k}$ block iteration configuration the number of iteration blocks the number of synchronization for each epoch is 12 , etc. The relationship between the number of block iterations and the computation time per epoch can be seen in Table 3.

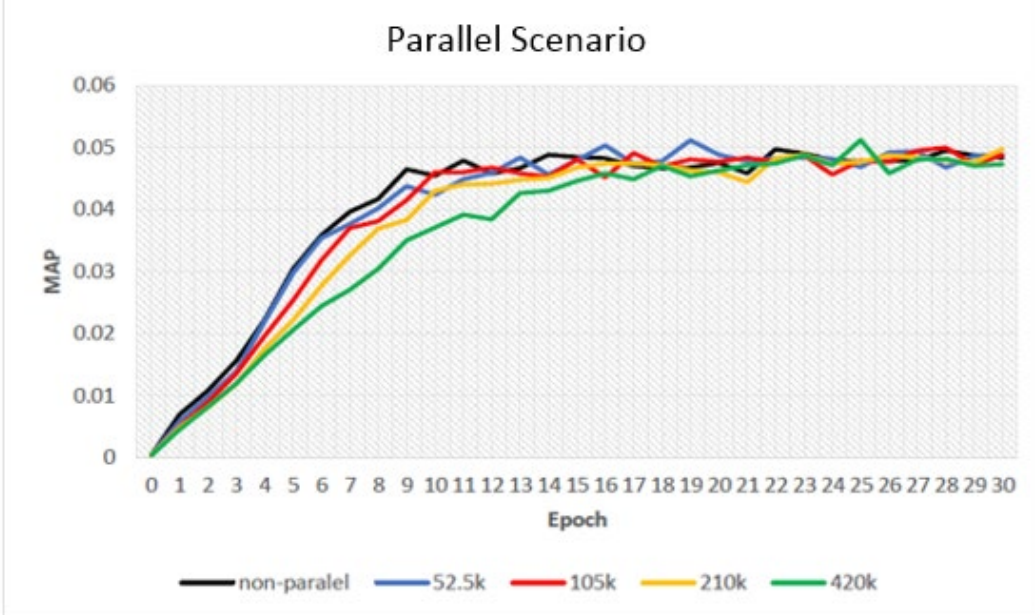

Figure 13. MAP as Function of Number of Iterations in Parallel BPR Scenario

Table 3. Computation Time In Parallel BPR Scenario

\begin{tabular}{|l|l|l|}
\hline Scenario/Configuration & $\begin{array}{l}\text { Computing time } \\
\text { (s/epoch) }\end{array}$ & $\begin{array}{l}\text { Number of } \\
\text { sync/epoch }\end{array}$ \\
\hline Non-paralel & 595 & 0 \\
\hline Paralel-420k blok iterasi & 335 & 3 \\
\hline Paralel-210k blok iterasi & 360 & 6 \\
\hline Paralel-105k blok iterasi & 397 & 12 \\
\hline Paralel-52,5k blok iterasi & 490 & 24 \\
\hline
\end{tabular}

From Table 3 above, the computation time required for each synchronization cannot be calculated with certainty because it has a different value for each configuration. For example in a $420 \mathrm{k}$ block iteration configuration, the time required for each synchronization is $(335-595 / 4)$ $/ 3=62$ seconds. Then in the $210 \mathrm{k}$ configuration, the time required for each sync is $(360-595 / 4)$ / $6=35.2$ seconds. The cause of this difference is still a question that has not been answered in this study. However, it can certainly be said that synchronization is the bottleneck of the LEARNBPR parallelization with the DSGD approach.

Furthermore, in Table 4 the total time required for each scenario / configuration is presented to achieve a convergent MAP. The optimum number of iteration blocks in this experiment is $105 \mathrm{k}$ with a total computation time of 6,749 seconds. The total non-parallel computation time is 8,330 seconds, in other words the increase achieved by parallel computing is $23 \%$ with 4 workers used. This increase was indeed insignificant compared to the number of workers used. As mentioned in the previous paragraph, the parallelization of LEARN BPR with the DSGD 
approach has a bottleneck in the synchronization process. The same thing was also stated in experiment [3] that the increase in computation speed with DSGD is not significant.

Table 4. Computation Time Relationship and Number of Iterations in Parallel Scenario

\begin{tabular}{|l|l|l|l|}
\hline Scenario/Configuration & $\begin{array}{l}\text { Computing time } \\
\text { (s/epoch) }\end{array}$ & Epoch & $\begin{array}{l}\text { Total Computing } \\
\text { Time }\end{array}$ \\
\hline Non-paralel & 595 & 14 & 8330 \\
\hline Paralel-420k block iters & 335 & 23 & 7705 \\
\hline Paralel-210k block iters & 360 & 22 & 7920 \\
\hline Paralel-105k block iters & 397 & 17 & 6749 \\
\hline Paralel-52,5k block iters & 490 & 14 & 6860 \\
\hline
\end{tabular}

In addition, the following is a comparison of the MAP value as a function of the epoch for LEARN-BPR parallelization with permutations and without permutations with the same configuration. From Figure 14 below, it is clear that there is a decrease in the MAP value if parallelization is carried out without matrix permutation.

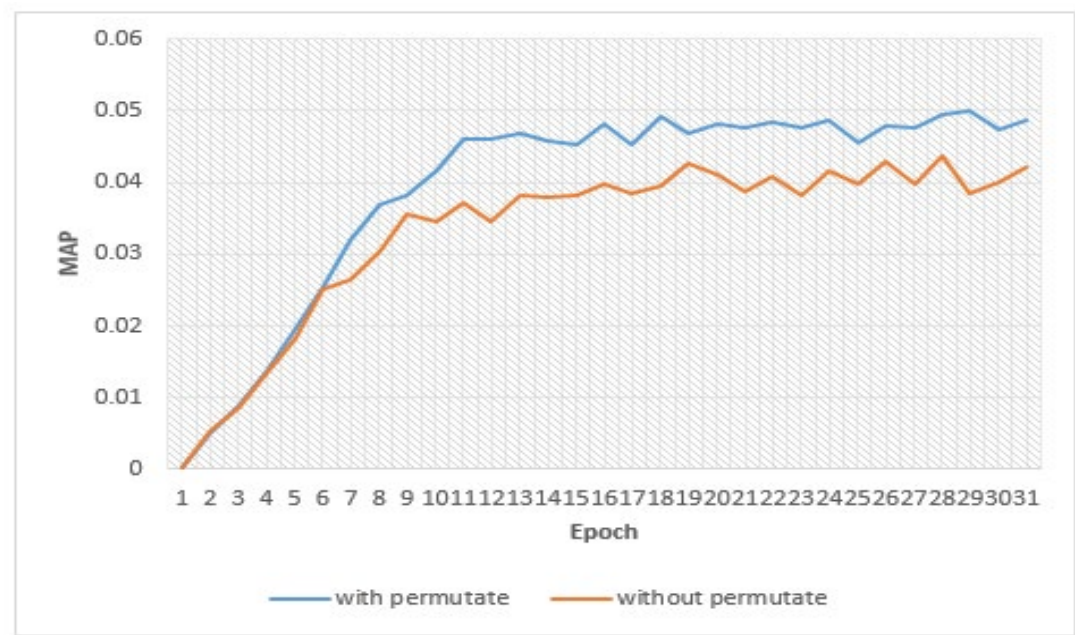

Figure 14. Comparison of MAP values as a function of epoch on LEARN-BPR parallelization with permutations and without permutations

\section{Related Work}

Recommender system has been widely researched from time to time in accordance with the challenges of the times. When the recommender system has begun to be widely applied to online platforms, the challenge that arises is how to design a large-scale recommender system. [12] won the Netflix price competition by proposing SVD, a large-scale recommender system based on matrix factorization. Furthermore, many other studies have tried to develop a matrix factorization-based recommender system. Furthermore, many other studies have tried to develop a matrix factorization-based recommender system. [13] succeeded in distributing computations in the matrix factorization model optimization process. This algorithm is known as Distributed Stochastic Gradient Descent (DSGD). On the other hand [14] proposed a matrix factorization model for implicit feedback. This approach is the development of SVD with confidence and L2 regularization. [5] developed Bayesian personalized ranking (BPR), an optimization framework for studying recommender systems from implicit feedback. This framework can be applied to the matrix factorization and KNN models. [11] tries to optimize the convergence rate in BPR with dynamic sampling strategy on negative items. Then [10] perfected this technique by 
modeling the relationship between the matrix factorization multiplication result and the sampling probability.

On the other hand, BPR have also been implemented in many cases, one of which is the streaming recommender system. [2] proposes a regularized kernel matrix factorization (RKMF) class that can be updated online without retraining the entire model. This approach has the advantage of a very fast model update rate. However, [3] proved that the online learning approach has a short term memory problem, namely the model tends to only keep track of recent activities, which causes the quality of predictions to be less accurate. They think that it is impossible to store all datasets in memory. Then they propose a reservoir approach to solve the short term memory problem in an online updating approach as well as solve the problem of limited computational memory. Reservoir is a representation of the sample stream at time $t$ and this reservoir has a fixed size. Model updating was carried out using data samples from selected reservoirs based on the principles of active learning. Furthermore, [4] argues that reservoir sampling tends to ignore current activities so that it cannot capture drift interest. Then they combined the reservoir data with the streaming window as input. Reservoir represents historical data and window represents current data. We expect that model can capture drift interest while maintaining long-term preferences. The method proposed in this study is adapting this method with modifications to the reservoir data selection technique. The reservoir data selection technique in this study was inspired by the dynamic oversampling technique proposed by Rendle.

\section{Discussion}

The recommender system designed in this research is expected to be an alternative method that can be used for the application of a recommender system in the real world. This method can be practically applied to recommender system applications, but at the application level it is necessary to design a recommender system mechanism. For example the division between the model update event and the recommendation event. In addition, a data stream handling mechanism is also needed and adjustments to the use of computing resources. There are several studies that focus on addressing this mechanism [15], [16].

In this study, experiments were carried out without using stream data sources and streaming data processing tools such as spark, kafka etc. However, the data source used is a dataset which is divided into windows. This is to simplify the settings during the experiment. However, the drawback of this testing mechanism is that the memory usage becomes excessive because all the data is stored in memory.

From a logical point of view, the designed LEARN BPR parallelization algorithm can be applied to distributed computing. Thus, the recommender system will be scaled up as needed and this algorithm can still work well with it. For example, when new users or items continue to increase and user activity increases so that the data stream rate is higher, so additional computing resources are needed. In addition to the reservoir approach, the size of the reservoir will affect the quality of the recommendations as shown in the experiment Diaz-Aviles et al.[3], but the addition of this reservoir size will increase the computation time. Parallel computing is also expected to provide room for increasing the size of the reservoir without causing overload.

\section{Conclusion}

In this research, the development of reservoir sample selection techniques at SPMF has been carried out. In addition, this research has also designed an algorithm for updating the SPMF model that can be computed in parallel. Based on the overall research results obtained in the previous chapters, it can be concluded that:

1. The selection of reservoir data samples using the weighted reservoir sampling technique is proven to be able to maintain the user's long-term interest more than the uniform reservoir sampling technique. Through the experiments conducted, the method designed is proven to produce better quality recommendations than the baseline method. In addition, the proposed method is also able to achieve at least $83 \%$ of the quality of BPR-MF with a reduction of the dataset by $78 \%$ and the computation time 60 times faster. 
2. DSGD can be implemented in LEARN BPR by inserting a permutation process for the arrangement of items in the matrix. The computational speed at LEARN BPR is affected by the number of synchronizations. The less number of synchronizations required, the shorter the computation time. Meanwhile, the number of synchronization needed to achieve convergence is influenced by the setting of the number of block iterations. In the experiment, the parallelization of LEARN BPR can increase computation speed by $23 \%$ by using 4 workers.

\section{Acknowledgement}

We thank Bukalapak for providing us with anonymized dataseset for our experimentation. We also thank Bukalapak-ITB Laboratorium for Artificial Intelligent dan Cloud Computing for supporting our research

\section{References}

[1] S. Chang et al., "Streaming recommender systems," 26th Int. World Wide Web Conf. WWW 2017, pp. 381-389, 2017.

[2] S. Rendle and L. Schmidt-Thieme, "Online-updating regularized kernel matrix factorization models for large-scale recommender systems," RecSys'08 Proc. 2008 ACM Conf. Recomm. Syst., pp. 251-258, 2008.

[3] E. Diaz-Aviles, L. Drumond, L. Schmidt-Thieme, and W. Nejdl, "Real-time top-n recommendation in social streams," 2012.

[4] W. Wang, H. Yin, Z. Huang, Q. Wang, X. Du, and Q. V. H. Nguyen, "Streaming Ranking Based Recommender Systems," in SIGIR '18: The 41st International ACM SIGIR Conference on Research \& Development in Information Retrieval, 2018, pp. 525-534.

[5] S. Rendle, C. Freudenthaler, Z. Gantner, and L. Schmidt-Thieme, "BPR: Bayesian personalized ranking from implicit feedback," Proc. 25th Conf. Uncertain. Artif. Intell. UAI 2009, pp. 452-461, 2009.

[6] P. S. Efraimidis, "Weighted random sampling over data streams," Lect. Notes Comput. Sci. (including Subser. Lect. Notes Artif. Intell. Lect. Notes Bioinformatics), vol. 9295, pp. 183$195,2015$.

[7] M. T. Chao, "A general purpose unequal probability sampling plan," Biometrika, vol. 69, no. 3, pp. 653-656, 1982.

[8] P. S. Efraimidis and P. G. Spirakis, "Weighted random sampling with a reservoir," Inf. Process. Lett., vol. 97, no. 5, pp. 181-185, 2006.

[9] J. S. Vitter, "Random Sampling with a Reservoir," ACM Trans. Math. Softw., vol. 11, no. 1, pp. 37-57, 1985.

[10] S. Rendle and C. Freudenthaler, "Improving Pairwise Learning for Item Recommendation from Implicit Feedback," pp. 273-282, 2014.

[11] W. Zhang, T. Chen, J. Wang, and Y. Yu, "Optimizing top-N collaborative filtering via dynamic negative item sampling," SIGIR 2013 - Proc. 36th Int. ACM SIGIR Conf. Res. Dev. Inf. Retr., pp. 785-788, 2013.

[12] Y. Koren, R. Bell, and C. Volinsky, "Matrix Factorization Techniques for Recommender Systems," IEEE, vol. 42, no. 8, pp. 30-37, 2009.

[13] R. Gemulla, P. J. Haas, E. Nijkamp, and Y. Sismanis, "Large-scale matrix factorization with distributed stochastic gradient descent," in Proceedings of the ACM SIGKDD International Conference on Knowledge Discovery and Data Mining, 2011, pp. 69-77.

[14] Y. Hu, C. Volinsky, and Y. Koren, "Collaborative filtering for implicit feedback datasets," Proc. - IEEE Int. Conf. Data Mining, ICDM, pp. 263-272, 2008.

[15] B. Chandramouli, J. J. Levandoski, A. Eldawy, and M. F. Mokbel, "StreamRec: A realtime recommender system," Proc. ACM SIGMOD Int. Conf. Manag. Data, pp. 1243-1245, 2011.

[16] C. A. Ludmann, M. Grawunder, and H. Jürgen Appelrath, “OdysseusRecSys: Collaborative 
filtering based on a data stream management system," CEUR Workshop Proc., vol. 1388, pp. 1-4, 2015.

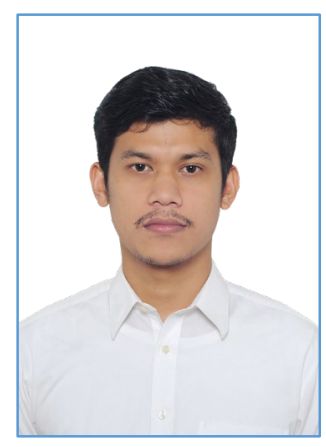

Berman Danyel Sinaga was born on Humbang Hasundutan, Indonesia. He received the bachelor degree of Petroleum Engineering from Institut Teknologi Bandung in 2016, and master degree in Informatics from Institut Teknologi Bandung in 2020. He is currently working as Data Engineer at IT's Buah, Indonesia. His research interests include machine learning, and business intelligence.

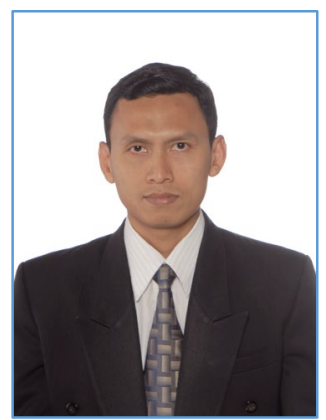

Saiful Akbar received the bachelor and master degrees in Informatics from the Department of Informatics, Institut Teknologi Bandung, Indonesia, in 1997 and 2002, respectively, and the doctorate degree in Engineering Science from Johannes Kepler University of Linz, Austria, in 2007. He was also a Visiting Researcher at Norwegian University of Science and Technology (NTNU) in 2009-2010. He is currently an assistant professor at School of Electrical Engineering and Informatics, Institut Teknologi Bandung. His research interests include data and knowledge engineering, multimedia database and similarity retrieval, information extraction, data analytics and visualization. 